\title{
Tracking pigeons in a magnetic anomaly and in magnetically "quiet" terrain
}

\author{
Ingo Schiffner • Patrick Fuhrmann • \\ Roswitha Wiltschko
}

Received: 29 March 2011 /Revised: 29 April 2011 / Accepted: 29 April 2011 /Published online: 21 June 2011

(C) The Author(s) 2011. This article is published with open access at Springerlink.com

\begin{abstract}
Pigeons were released at two sites of equal distance from the loft, one within a magnetic anomaly, the other in magnetically quiet terrain, and their tracks were recorded with the help of GPS receivers. A comparison of the beginning of the tracks revealed striking differences: within the anomaly, the initial phase lasted longer, and the distance flown was longer, with the pigeons' headings considerably farther from the home direction. During the following departure phase, the birds were well homeward oriented at the magnetically quiet site, whereas they continued to be disoriented within the anomaly. Comparing the tracks in the anomaly with the underlying magnetic contours shows considerable differences between individuals, without a common pattern emerging. The differences in magnetic intensity along the pigeons' path do not differ from a random distribution of intensity differences around the release site, indicating that the magnetic contours do not directly affect the pigeons' routes. Within the anomaly, pigeons take longer until their flights are oriented, but $5 \mathrm{~km}$ from the release point, the birds, still within the anomaly, are also significantly oriented in the home direction. These findings support the assumption that magnetically anomalous conditions initially interfere with the pigeons' navigational processes, with birds showing rather individual responses in their attempts to overcome these problems.
\end{abstract}

Communicated by: Sven Thatje

Electronic supplementary material The online version of this article (doi:10.1007/s00114-011-0802-3) contains supplementary material, which is available to authorized users.

I. Schiffner $\cdot$ P. Fuhrmann $\cdot$ R. Wiltschko $(\bowtie)$

Fachbereich Biowissenschaften der J.W.Goethe-Universität

Frankfurt,

Siesmayerstraße 70,

60054 Frankfurt am Main, Germany

e-mail: wiltschko@bio.uni-frankfurt.de
Keywords Magnetic anomaly P Pigeon navigation . Homing · GPS tracking · Magnetic "map" factors . Point of Decision

\section{Introduction}

The ability of birds, in particular of homing pigeons, to return from distant, unknown sites is well documented (see Wiltschko 1992 for a summary of displacement experiments with wild birds); the factors they use to determine their home direction, however, are not yet known with certainty. A multitude of factors have been suggested, among them are magnetic cues (Viguier 1882; Wagner 1976; Walcott 1978), Coriolis force (Yeagley 1947), olfactory cues (e.g., Papi et al. 1971; Papi 1986; Gagliardo et al. 2009; but see Jorge et al. 2009, 2010), infrasound (Quine 1982; Hagstrum 2000), gravity (Lednor and Walcott 1984), and landscape features (see Baker 1984), but only few are supported by experimental evidence, and their specific role in the multi-modal navigational "map" of birds (Keeton 1974; Walcott 2005) remains largely unclear.

For magnetic cues, the behavior of homing pigeons released within magnetic anomalies, i.e., in areas with magnetic intensities deviating markedly from the regular regional field because of variation in the magnetic properties of underground rock formations, has always been important evidence for the use of magnetic factors in avian navigation. Wagner (1976) and Frei and Wagner (1976) were the first to conduct systematic studies in weak magnetic anomalies in Switzerland; they reported large deviations from the home direction associated with the direction of magnetic gradients. Walcott (1978), Kiepenheuer (1982), Lednor and Walcott (1988), and Wiltschko et al. (2009, 2010), releasing pigeons in stronger, highly irregular anomalies in the northeastern 
USA and Germany, observed a marked increase in scatter, leading to disorientation when the differences in magnetic intensity and/or the steepness of magnetic gradients exceeded a certain amount. Short vectors and prolonged vanishing intervals suggested that pigeons had problems with navigation. Most of these studies were traditional releases based on the visual observation of vanishing bearings about 2 to $2.5 \mathrm{~km}$ from the release point. The only exception was Walcott (1978) who released pigeons with small transmitters, tracked them by airplane, and documented these tracks to about $18 \mathrm{~km}$ from the release point. Yet, these tracks were rather crude, showing little detail on how the pigeons responded to the local conditions.

The availability of modern GPS techniques allows us to record the pigeons' routes with great accuracy and remarkable detail. Dennis and colleagues (2007) were the first to take advantage of GPS-based recorders in a study on the effects of magnetic anomalies. Analyzing the tracks of pigeons released within a moderately strong, fairly regular anomaly in New Zealand, they described phenomena like flying along magnetic isolines or gradients, i.e., pigeons choosing routes that follow lines of equal magnetic intensity and routes that coincide with the steepest change in magnetic intensity, but give few quantitative data. The observed alignments seem to be only of a transient nature, and their biological significance is not obvious. The few examples given do not allow a clear picture of the actual behavior to emerge.

In the present study, we released pigeons at two sites, one within the rather irregular magnetic Vogelsberg anomaly, the other in a magnetically "quiet" region, and recorded their tracks with GPS-based flight recorders. Our analysis focuses on the comparison of the behavior at the beginning of the homing flight, because within the Vogelsberg anomaly, our pigeons appear to give up magnetic cues in favor of other, non-magnetic factors before leaving the anomaly (Wiltschko et al. 2009, 2010).

\section{Materials and methods}

The experimental releases were performed in August 2009 and June and July 2010, following a small pilot study in August 2007 and May 2008, with the three tracks of this pilot study included in the analysis.

\section{Release sites}

We used two release sites situated in about equal distance in almost opposite directions; both sites lay in rural regions, with the nearest village in similar distances and directions from the home direction:
1. Oberlais $\left(50^{\circ} 24^{\prime} \mathrm{N}, 9^{\circ} 06^{\prime} \mathrm{E}\right), 44.8 \mathrm{~km}$ northeast of the loft; home direction, $225^{\circ}$; within the Vogelsberg anomaly, an area with strong, irregular fluctuations in intensity and steep local magnetic gradients in varying directions, with the release point about $10 \mathrm{~km}$ from the edge of the anomaly in the homeward direction. At the release point, the intensity was $133 \mathrm{nT}$ above the one at the loft.

2. Essenheim $\left(49^{\circ} 47^{\prime} \mathrm{N}, 8^{\circ} 08^{\prime} \mathrm{E}\right), 42.2 \mathrm{~km}$ southwest of the loft; home direction, $61^{\circ}$; the control site in magnetically "quiet" terrain, where the magnetic intensity changes very little in all directions; at the release point, it was $5 \mathrm{nT}$ above the one at the loft.

The local magnetic conditions around the two sites are illustrated in Fig. 1, based on magnetic intensity data for $100 \times$ $100 \mathrm{~m}$ squares showing the deviations from the reference field (DGRF 1980.0) provided by the Leibniz Institute of Applied Geophysics. The two sites are identical to the sites A2 and C2 of our previous study (Wiltschko et al. 2010).

\section{Test birds}

The test birds were adult pigeons from our Frankfurt loft $\left(50^{\circ} 08^{\prime} \mathrm{N}, 8^{\circ} 40^{\prime} \mathrm{E}\right)$. The magnetic conditions at the loft, $57 \mathrm{nT}$ below the reference field in a magnetic minimum, are given in detail in Wiltschko and Wiltschko (2003a). The birds were at least 1 year old. In their first year of life, they had completed a standard training program up to $40 \mathrm{~km}$ in the cardinal compass directions and had been additionally released for several training flights up to $30 \mathrm{~km}$ in spring each year. All had completed additional training flights up to $20 \mathrm{~km}$ carrying dummy weights to prepare them for carrying the GPS recorder, had participated in previous tracking experiments from various sites, and thus had ample experience in flying with the recorder. In the present study, all pigeons were unfamiliar with the release sites, i.e., they had never homed from these specific sites before.

\section{GPS tracking devices}

The GPS recorders used in this study were based on the prototype developed by von Hünerbein et al. (2000), with either an embedded patch antenna or a Y-antenna and a data logger as additional components. The weight including the battery ranged from $35 \mathrm{~g}$ in the pilot study to $23 \mathrm{~g}$ used later. The recorder was set to take a positional fix every second, with a precision of $\pm 4 \mathrm{~m}$ in the older models and $\pm 1.8 \mathrm{~m}$ in the recent models. After the receivers had contact with a sufficient number of satellites, they were wrapped in plastic to shield them from water and attached to the pigeons' backs by means of a harness made from Teflon tape (see Wiltschko et al. 2007 for details). Immediately before release, the 

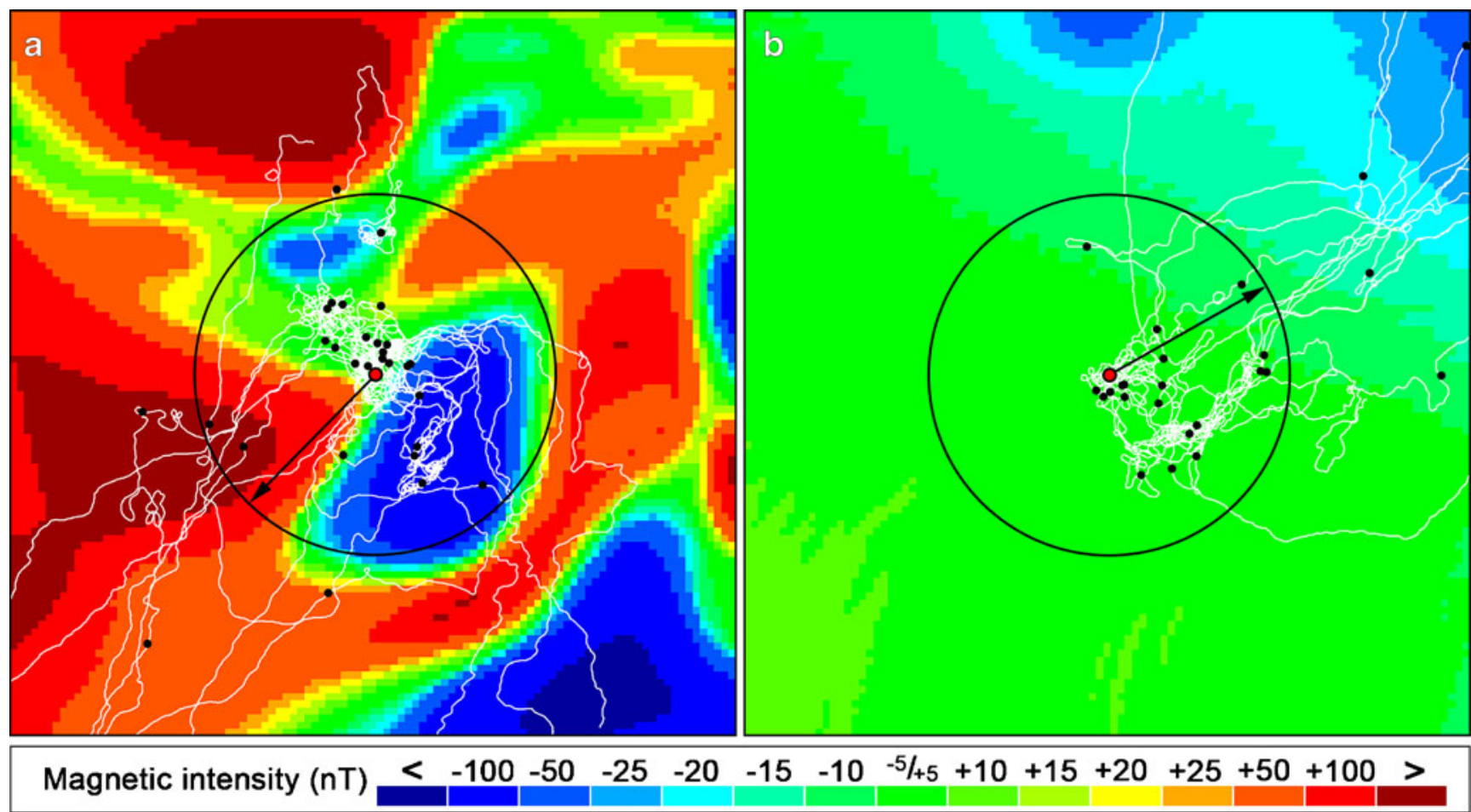

Fig. 1 Magnetic conditions and tracks within an area of $5 \times 5 \mathrm{~km}$ around the release sites: Oberlais within the anomaly (a) and Essenheim within magnetically "quiet" terrain (b). The colors in the map indicate the differences in intensity (given in $100 \times 100 \mathrm{~m}$ squares) to those at the

recorder was placed on the dorsal plate of the harness and fixed with Velcro and additional sticky tape. The harness and coating added another $7 \mathrm{~g}$ to the load.

From 18 pigeons released within the anomaly and 17 pigeons released at the control site, we obtained 12 tracks and 13 evaluable tracks, respectively; the others had to be excluded from analysis because of recorder failure or pigeons flying only short distances $(<3 \mathrm{~km})$ and returning only after the battery had run empty.

Track analysis and statistics

In the present study, we focus on the beginning of the flight within $5 \mathrm{~km}$ from the release point. In a first step, we compared the behavior before and after the first "Points of Decision." This first Point of Decision is defined by the highest increase in the steadiness of flight; it marks the moment when the pigeon begins to leave the release site, with steadiness and flying speed increasing. For identifying a Point of Decision, steadiness is determined as sliding means of the vector length calculated every $15 \mathrm{~s}$ from 60 consecutive headings of the tracked pigeon, with the headings being the direction between two consecutive positional fixes (Schiffner and Wiltschko 2009 for details). The first Point of Decision, usually close to the release point, separates the initial phase of flying around at the release site from the following departure phase. Over longer distances, release point - note that the scale is non-linear. The release sites in the center of a circle with the radius $2.5 \mathrm{~km}$ is marked by a red dot; the arrow indicates the home direction. The tracks are shown as white lines, with the black dots marking the Points of Decision

most tracks include periods where the pigeons fly steadily towards home and periods where they do not increase the distance from the release site continuously. Hence, additional Points of Decision can be defined by the highest increase in steadiness following these stalling periods, using the method described above (see Schiffner et al. 2011).

We determined the positions of the first "Point of Decision" of all tracks from each site and calculated (1) their center of distribution and (2) their median distance from the release point. For the initial phase of each track, we determined (3) its duration, (4) the distance flown, (5) the mean vector of headings, based on all headings during this phase, (6) the absolute deviations of the mean heading from the home direction, and (7) the steadiness of flight, now represented by the mean vector lengths resulting from all headings of the track during the entire duration of the specific phase. The mean vector, the absolute deviation from the home direction, and the steadiness were also calculated for the departure phase, excluding data beyond $5 \mathrm{~km}$ from the release point.

From the data for the individual pigeons, we then determined second order means for the mean headings and the medians of the other variables for the tracks from both sites. In addition, we calculated mean vectors from the bearings 2.5 and $5 \mathrm{~km}$ from the release point. These variables were then compared between the two release sites. 
The distribution of the first Points of Decision were tested with Hotelling's one-sample test for bivariate samples for directional preferences and compared between the two sites with Hotelling's two-sample test. The second order mean vectors were tested for directional preference with the Rayleigh test and compared using the Mardia Whatson Wheeler test (Baschelet 1981). The other variables were compared using the Mann-Whitney $U$ test.

In a second step, we analyzed the relationship of the individual tracks with the local distribution of magnetic intensity, in particular with the changes in intensity the pigeons experienced in the course of their flight. We recorded the difference in intensity between the consecutive $100 \times 100 \mathrm{~m}$ squares a pigeon crossed, and calculated, for each track, (1) the maximum difference in total intensity the pigeon had experienced during the first minute of its flight and (2) the percentage of the various differences among the total differences between all segments the pigeons had visited until $5 \mathrm{~km}$ from the release point. For comparison, we generated two sets of random distributions derived from the local distribution of magnetic intensity, the first by determining the differences based on 12 trajectories, defined as straight lines $30^{\circ}$ apart originating at the release point and ending at a distance of $5 \mathrm{~km}$ starting at the north and the second by 13 similar trajectories $15^{\circ}$ apart within the homeward semicircle. The distribution of differences in the pigeons' tracks and the random distributions were then compared with two-way ANOVA with repeated measurements. This analysis was performed only for the anomaly data because, in the magnetically quiet region around the control site, the differences in intensity, mostly being 0 or $1 \mathrm{nT}$ and never exceeding $2 \mathrm{nT}$, were too small for this type of approach.

\section{Results}

Our analysis is based on 13 tracks from Oberlais within the magnetic anomaly and 12 tracks from the control site Essenheim within magnetically quiet terrain. Fig. $1 \mathrm{a}, \mathrm{b}$ shows the tracks within $5 \mathrm{~km}$ from the two release sites on maps indicating the local magnetic intensity (see Fig. S1 in Electronic Supplementary Material for better resolution). Figures S2 and S3 in ESM give the same tracks on topographical maps indicating villages, roads, forests, etc. and on maps indicating the altitude of the terrain. Table 1 compares the data calculated from the beginning of the tracks, with Table S1 in the ESM giving the data for each track separately.

The behavior of pigeons within the anomaly differed significantly from that of pigeons at the control site in several aspects. From both release sites, pigeons headed out in various directions, and they made their first decision at similar distances from the release site. However, the increased time and the extended distance traveled within the anomaly show that the pigeons took considerably longer before their first Point of Decision (see Table 1). During the initial phase, the steadiness of flying was

Table 1 Comparing variables from the beginning of the tracks between the two sites (mean vectors and medians given)

\begin{tabular}{|c|c|c|c|c|}
\hline $\begin{array}{l}\text { Variable (medians given) } \\
N, \text { number of tracks }\end{array}$ & $\begin{array}{l}\text { Control site } \\
13\end{array}$ & $\begin{array}{l}\text { Anomaly site } \\
12\end{array}$ & $\begin{array}{l}\text { Testing for } \\
\text { difference }\end{array}$ & \\
\hline First Points of Decision, center & $+35^{\circ}, 498 \mathrm{~m}^{*}$ & $+100^{\circ}, 597 \mathrm{~m}^{*}$ & $F_{2,24}=3.9$ & n.s. \\
\hline \multicolumn{5}{|c|}{ Initial phase before the first Point of Decision: } \\
\hline Duration of initial phase & $105 \mathrm{~s}$ & $225 \mathrm{~s}$ & $U_{\mathrm{M}}=47.0$ & $p<0.05$ \\
\hline Distance flown & $1,405 \mathrm{~m}$ & $3,694 \mathrm{~m}$ & $U_{\mathrm{M}}=44.0$ & $p<0.05$ \\
\hline Mean vector of headings & $+45^{\circ}, 0.65^{* *}$ & $-176^{\circ}, 0.48^{\mathrm{n} . \mathrm{s}}$ & $U_{\mathrm{C}}=7.5$ & $p<0.05$ \\
\hline Absolute deviation from home & $55^{\circ}$ & $143^{\circ}$ & $U_{\mathrm{M}}=26.5$ & $p<0.01$ \\
\hline Steadiness of flight & 0.29 & 0.18 & $U_{\mathrm{M}}=51.0$ & $p<0.10$ \\
\hline \multicolumn{5}{|c|}{ Departure phase after the first Point of Decision: } \\
\hline Mean vector of headings & $+19^{\circ}, 0.74^{* * *}$ & $-43^{\circ}, 0.23^{\text {n.s. }}$ & $U_{\mathrm{C}}=5.75$ & $p<0.10$ \\
\hline Absolute deviation from home & $45^{\circ}$ & $77^{\circ}$ & $U_{\mathrm{M}}=50.5$ & $p<0.10$ \\
\hline Steadiness of flight & 0.75 & 0.75 & $U_{\mathrm{M}}=75.0$ & n.s. \\
\hline $2.5 \mathrm{~km}$ from the release point & $+7^{\circ}, 0.86^{* * *}$ & $-32^{\circ}, 0.39^{\text {n.s. }}$ & $U_{\mathrm{C}}=2.37$ & n.s. \\
\hline $5.0 \mathrm{~km}$ from the release point & $+12^{\circ}, 0.93^{* * *}$ & $-21^{\circ}, 0.85^{* * *}$ & $U_{\mathrm{C}}=1.64$ & n.s. \\
\hline
\end{tabular}

Directions are given as deviations from the respective home direction, with + indicating a clockwise and - a counter-clockwise deviation

$F_{2,24}$ test statistic of the Hotelling two-sample test, $U_{M}$ test statistic of the Mann-Whitney test, $U_{C}$ test statistic of the Mardia Watson Wheeler test for circular distributions (Baschelet 1981). Asterisks at the center of the first Point of Decision and at mean vectors indicate significant differences from random (Hotelling's one-sample test and Rayleigh test, respectively). Significance levels: ${ }^{*} \mathrm{p}<0.05, * * \mathrm{p}<0.01, * * * \mathrm{p}<0.001 ; n . s$. not significant 
generally rather low, but it tended to be lower within the anomaly. The distributions of the mean headings differed, with the pigeons in magnetically quiet terrain significantly oriented and those within the anomaly deviating significantly farther from the home direction.

During the departure phase after the first Point of Decision, the pigeons released in magnetically quiet terrain were already fairly well oriented towards home, deviating only $19^{\circ}$ from the home direction, whereas the pigeons released within the anomaly were not yet significantly oriented and still tended to show a larger deviation from the home direction. The steadiness of flight increased at both sites and did not differ any longer. Several birds have a second Point of Decision near the release site; in the following phase, the birds in magnetically quiet terrain are well oriented towards home ( $n=6,-4^{\circ}, 0.93 ; p<0.01$, Rayleigh test), whereas the birds within the anomaly are still not significantly oriented $(n=$ $\left.7,+95^{\circ}, 0.40 ; p>0.05\right)$.

The tracks within the anomaly are shown in Fig. 1a, and those in the magnetically quiet region, in Fig. 1b. At both sites, there are considerable differences between individuals, but while at the control site, the tracks largely stayed within the homeward semicircle; they covered a considerably larger area within the anomaly. The maximum differences in magnetic intensity experienced during the first minute of flight in the anomaly, between 8 and $33 \mathrm{nT}$ (median, $18 \mathrm{nT}$ ), are significantly larger than those experienced at the control site, between 0 and $4 \mathrm{nT}$ (median, $2 \mathrm{nT} ; U=0, p<0.001$, Mann Whitney $U$ test). Some pigeons showed a tendency to head towards the nearest village, which is reflected by the centers of the Points of Decision shifting in the respective directions (see Fig. S2a, b in ESM). Thereafter, the pigeons at the control site are fairly well oriented towards home, whereas those

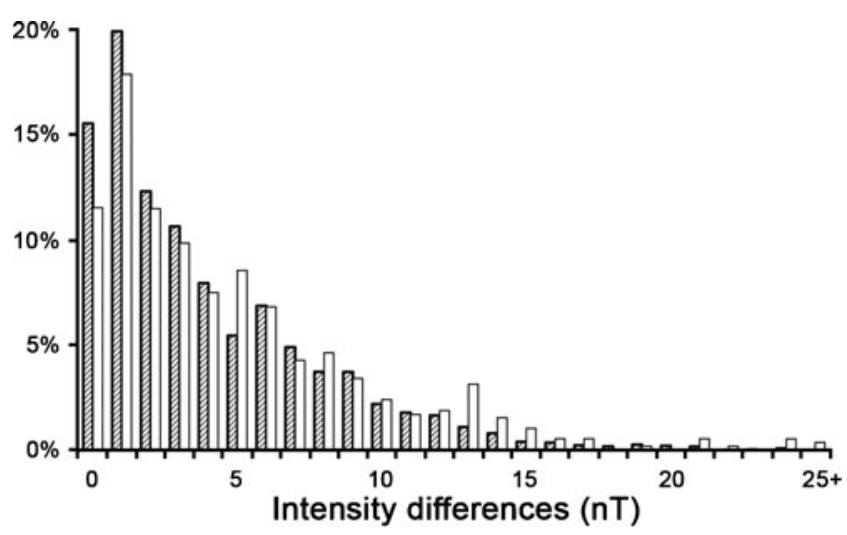

Fig. 2 Distribution of differences encountered by the pigeons along their routes through the anomaly until they passed the 5-km line (hatched bars) compared to those of a random distribution generated on the basis of 12 trajectories, one every $30^{\circ}$, from the release point to the 5-km line (open bars) released within the anomaly continued to head in different directions (Table 1), a behavior that is not due to constraints of the local topography (see Fig. S3a in ESM). While some pigeons soon depart from the area, heading more or less towards home, others spend an extended time flying about. Yet, finally, they, too, leave the area, and $5 \mathrm{~km}$ from the release point, still well within the anomaly, the pigeons are en route, oriented towards home (see Table 1).

Looking for patterns in the tracks, we compared the differences in magnetic intensity the pigeons experienced when entering the next $100 \times 100 \mathrm{~m}$ squares with two random distributions of magnetic intensity generated from the local magnetic situation. Within the anomaly, pigeons fly in about two thirds of all cases along routes where the difference to the previous square is less than $5 \mathrm{nT}$ (Fig. 2). However, the magnetic intensity changes along these routes are not significantly different from the changes along routes randomly distributed around the release site $\left(F_{1,25}=0.0000\right.$, $p=0.9955$, ANOVA) or within the homeward semicircle $\left(F_{1,25}=0.0002, p=0.9894\right.$, ANOVA; see Fig. S4 in ESM $)$. This means that the routes of the pigeons do not show any specific pattern with respect to the local magnetic contours.

\section{Discussion}

Previous traditional studies in the Vogelsberg anomaly (Wiltschko et al. 2009, 2010) had suggested an effect of the anomalous conditions which can now be analyzed in detail based on GPS-recorded tracks: while a certain amount of flying around somewhat erratically immediately after being released and heading towards villages is a typical behavior also occurring at the control site and thus not related to the magnetic situation, the increase in scatter and the longer vanishing intervals within the magnetic anomaly observed in the traditional studies, which were found to be correlated with the magnetic variance at the sites (see Wiltschko et al. 2009, 2010), seem to be a response to experiencing unusually large and rapid changes in magnetic intensity, reflecting the pigeons' confusion. It tends to decrease the steadiness and goes hand in hand with an increase in the time taken and distance flown before the pigeons make a decision to leave the site. This is a clear indicator that the long vanishing times within the anomaly (Wiltschko et al. 2009, 2010) are not a result of decreased motivation, but rather a direct effect of the irregular magnetic conditions.

After the first Point of Decision, the behavior of the pigeons within the anomaly shows considerable individual variation: some birds seem to abandon the magnetic field as a navigational factor and leave the site, probably guided by non-magnetic cues, while others seem to be still confused and continue to fly about erratically. But in the end, the pigeons within the anomaly are also more or less homeward 
oriented $5 \mathrm{~km}$ from the release point. Initial disorientation is just a transient effect, limited to the area around the release point. This contrasts with the findings of Walcott (1978) who reported randomly oriented transmitter bearings about $18 \mathrm{~km}$ from the release point. It appears to reflect the different situation, in particular concerning the availability of non-magnetic cues, in the two anomalies (see Wiltschko et al. 2010).

Another important aspect of our study is that we do not find any indications for specific behavioral patterns with relation to the changes in magnetic intensity. When looking at the tracks, one can find sections of routes that could be interpreted as suggesting previously described behaviors such as flying along the steepest magnetic gradients or following isolines, i.e., lines of equal intensity, as reported by Wagner (1976), Frei and Wagner (1976) or, more recently, by Dennis and colleagues (2007) for the beginning of the pigeons' flight. Yet, these are transient and concern only rather short portions of the tracks. Pigeons are not able to actually perceive magnetic gradients per se or determine the direction of isolines directly - the magnetic sense cannot perceive distant changes like, e.g., the eyes, but can only measure the magnetic intensity at the pigeon's current position and record changes in intensity. For detecting gradient directions or the course of isolines, pigeons would have to scan the respective area. For a direct response to local magnetic contours, we can define clear expectations: if pigeons preferably flew along magnetic gradients, we would have expected them to avoid flying into areas with no or only little changes in intensity. On the other hand, if they preferred to follow isolines, we would expect them to avoid flying into areas with greater changes in intensity. We find that in about two thirds of the cases the birds fly into squares with little intensity differences. However, the observed changes along the tracks do not differ from the random data sets, pointing out that the pigeons do not show a preference for either type of behavior. The sections of the tracks that coincide with isolines or gradient directions seem to do so by chance. This suggests that the specific routes chosen by the pigeons are not directly guided by the local magnetic contours.

This seems to be at variance with the observations by Dennis and colleagues (2007) who described following isolines or flying along gradients from tracks in an anomaly in New Zealand and claimed that almost two thirds of the tracks showed significantly aligned segments, some only over rather short distances, others over longer distances. The basis of their calculations for random probability is not given in detail, and it is not clear whether they took the local conditions at their release sites into account. In the few examples given, extensive alignments are not necessarily obvious. We, too, observe short sections of the tracks coinciding with magnetic isolines or gradients, but altogether, we cannot confirm their observations, as our pigeons encounter the changes in intensity that are expected by flying randomly with regard to the magnetic contours. Following isolines or gradients thus does not seem to be general features of the pigeons' behavior within magnetic anomalies. It has to be noted, however, that the anomaly in New Zealand where Dennis and colleagues (2007) performed their study appears to be much more regular than the erratic Vogelberg anomaly with its rapid, irregular changes in intensity and steep gradient of varying directions - here, neither following isolines nor flying along magnetic gradients would aid the pigeons to find their way out of the anomaly.

The general concept of pigeon navigation (Wallraff 1974; Wiltschko and Wiltschko 2003b) assumes that birds derive their home course by comparing local gradient values with the values at their home loft. In view of this, the behavior observed within the anomaly is easily explained: when trying to obtain the local intensity value for the navigational process, the pigeons experience large and rapid changes in magnetic intensity that do not provide a meaningful baseline for the comparison of magnetic values. Confused, they fly around erratically, until after a while, which differs greatly between individuals, they realize that the magnetic factors are unreliable and give them up in favor of non-magnetic cues, which then guide their homing flight. The nature of these alternative cues is still unclear, and it is far beyond the scope of the present study to determine their nature; they might be landscape features (Baker 1984), infrasound (Hagstrum 2000), olfactory cues (Papi 1986: but see Jorge et al. 2010), or others, even ones not yet considered. Disorientation as observed in earlier studies in anomalies (Walcott 1978; Kiepenheuer 1982) also occurs in our present study, but is clearly a transient effect in the very beginning of the flight. In the Vogelsberg anomaly, suitable non-magnetic factors seem readily available - this is indicated by the finding that pigeons deprived of magnetic input show oriented behavior and shorter vanishing intervals than untreated control birds (Wiltschko et al. 2010) and also, in the present study, by the observation that the pigeons are fairly well homeward oriented before they have left the magnetic anomaly.

Acknowledgments Our work was supported by the Deutsche Forschungsgemeinschaft (grants to RW). We thank K. Schäfer and B. Siegmund for their help with releasing the pigeons. Thanks are also due to the Leibniz Institute for Applied Geophysics, Hannover, Germany, for providing the data on magnetic intensity. The experiments were performed in accordance with the rules and regulations of animal welfare in Germany.

Open Access This article is distributed under the terms of the Creative Commons Attribution Noncommercial License which permits any noncommercial use, distribution, and reproduction in any medium, provided the original author(s) and source are credited. 


\section{References}

Baschelet E (1981) Circular statistics in biology. Academic, London

Baker R (1984) Bird navigation: the solution of a mystery? Holmes and Meier, New York

Dennis TE, Rayner MJ, Walker MM (2007) Evidence that pigeons orient to geomagnetic intensity during homing. Proc R Soc B 274:1153-1158

Frei U, Wagner G (1976) Die Anfangsorientierung von Brieftauben im erdmagnetisch gestörten gebiet des Mont Jurat. Rev Suisse Zool $83: 891-897$

Gagliardo A, Ioalè P, Savini M, Wild JM (2009) Navigational abilities of adult and experienced pigeons deprived of olfactory or trigeminally mediated magnetic information. J Exp Biol 212:3119-3124

Hagstrum JT (2000) Infrasound and the avian navigational map. J Exp Biol 203:1103-1111

Jorge PE, Marques AE, Phillips JB (2009) Activational rather than navigational effects of odors on pigeon homing. Curr Biol 19:650 654

Jorge PE, Marques AE, Phillips JB (2010) Activational effects of odours on avian navigation. Proc R Soc B 277:45-49

Keeton WT (1974) The orientational and navigational basis of homing in birds. Adv Study Behav 5:47-132

Kiepenheuer J (1982) The effect of magnetic anomalies on the homing behavior of pigeons. In: Papi F, Wallraff HG (eds) Avian navigation. Springer, Berlin, pp 120-128

Lednor AJ, Walcott C (1984) The orientation of pigeons at gravity anomalies. J Exp Biol 111:259-263

Lednor AJ, Walcott C (1988) Orientation of homing pigeons at magnetic anomalies: the effect of experience. Behav Ecol Sociobiol 22:3-8

Papi F, Fiore L, Fiaschi V, Benvenuti S (1971) Olfaction and homing in pigeons. Monit Zool Ital 6:85-95

Papi F (1986) Pigeon navigation: solved problems and open questions. Monit Zool Ital 20:471-517

Quine DB (1982) Infrasounds: a potential navigational cue for homing pigeons. In: Papi F, Wallraff HG (eds) Avian navigation. Springer, Berlin, pp 373-376
Schiffner I, Wiltschko R (2009) Point of Decision: when do pigeons decide to head home? Naturwissenschaften 96:251-258

Schiffner I, Pavkovic T, Siegmund B, Wiltschko R (2011) Strategies of young pigeons during 'map' learning. J Navig 64(3)

Viguier C (1882) Le sens de l'orientation et es organes chez las animaux et chez 1'homme. Rev Phil France Etranger 14:136

von Hünerbein K, Hamann HJ, Rüter E, Wiltschko W (2000) A GPS-based system for recording the flight paths of birds. Naturwissenschaften $87: 278-279$

Wagner G (1976) Das Orientierungsverhalten von Brieftauben im erdmagnetisch gestörten Gebiet des Chasseral. Rev Suisse Zool $83: 883-890$

Walcott C (1978) Anomalies in the earth's magnetic field increase the scatter of pigeons' vanishing bearings. In: Schmidt-Koenig K, Keeton WT (eds) Animal migration, navigation and homing. Springer, Heidelberg, pp 143-151

Walcott C (2005) Multi-modal orientation cues in homing pigeons. Integr Comp Biol 45:574-581

Wallraff HG (1974) Das Navigationssystem der Vögel. Schriftenreihe 'Kybernetik'. R. Oldenbourg Verlag, München, Wien

Wiltschko R (1992) Das Verhalten verfrachteter Vögel. Vogelwarte 36:249-310

Wiltschko R, Wiltschko W (2003a) Orientation behavior of homing pigeons at the Gernsheim anomaly. Behav Ecol Sociobiol 54:562-572

Wiltschko R, Wiltschko W (2003b) Avian navigation: from historical to modern concepts. Anim Behav 65:257-272

Wiltschko R, Schiffner I, Siegmund B (2007) Homing flights of pigeons over familiar terrain. Anim Behav 74:12291240

Wiltschko R, Schiffner I, Wiltschko W (2009) A strong magnetic anomaly affects pigeon navigation. J Exp Biol 212:29832990

Wiltschko R, Schiffner I, Fuhrmann P, Wiltschko W (2010) The role of the magnetite-based receptors in the beak in pigeon homing. Curr Biol 20:1534-1538

Yeagley HL (1947) A preliminary study of a physical basis of bird navigation. J Appl Phys 18:1035-1063 